\title{
Letters
}

\section{The significance of phosphotyrosine phosphatase (PTPase) 1B in insulin signalling}

\begin{abstract}
Dear Sir,
Just recently, a paper on insulin sensitivity in phosphotyrosine phosphatase (PTPase) 1B knock-out mice was published [1]. The importance of the paper was highlighted in a news report in the British Medical Journal [2]. The paper reported that knock-out mice grew normally and were fertile. Blood glucose and insulin were lowered and insulin sensitivity and insulin induced phosphorylation of the insulin receptor in muscle and liver from PTPase 1B -/-mice were enhanced. Moreover, the PTPase IB knock-out mice were resistant to weight gain and remained insulin sensitive on a high-fat diet. The findings confirmed that PTPase $1 \mathrm{~B}$ is an important enzyme in the regulation of intracellular insulin receptor signalling, and thereby a potential target in the treatment of Type II (non-insulin-dependent) diabetes mellitus and obesity [1].

After the description of the regulatory function of PTPases on intracellular tyrosine kinase signalling chains, the hypothesis was advanced that PTPase activity could decrease the intracellular amplification in the insulin cascade and that an increased PTPase activity or concentration or both could confer insulin resistance. The hypothesis was supported by vanadate restoring hyperglycaemia in several animal models of diabetes to normal. Later it was described that vanadate also increased insulin sensitivity in Type II diabetic patients. In accordance with the hypothesis, an increase in basal PTPase activity in a muscle fraction was found in 1991 in 8 insulin resistant patients (7 were Pima Indians) [3]. The authors suggested that the results could reflect a primary defect in Type II diabetes [3]. The findings gave birth to the idea that Type II diabetes could be treated medically with PTPase inhibitors to overcome the direct cause of the weakened intracellular insulin signal and thus increase glucose uptake in peripheral tissue. For optimal treatment it needed to be investigated whether a PTPase with specificity for the insulin receptor or other proteins or both in
\end{abstract}

Corresponding author: D. Worm, MD, PhD, Department of Medical Physiology, Bldg. 12, 6th floor, The Panum Institute, University of Copenhagen, Blegdamsvej 3C, DK-2200 Copenhagen N, Denmark the insulin-induced cascade existed and, if so, how it could be selectively inhibited.

Phosphotyrosine phosphatase 1B inhibits insulin signalling in cell systems. Furthermore, PTPase IB protein and PTPase activity was increased in skeletal muscle fractions from certain rodent models of insulin resistance, suggesting an important role of PTPase 1B in the development of this syndrome. The recent results on PTPase 1B knock-out mice extend these findings to an in vivo situation [1].

It is, however, impossible to account for most of the studied forms of insulin resistance by a simple model in which increased skeletal muscle PTPase activity or PTPase IB protein or both is responsible for decreased insulin signalling. Actually, several studies describe decreased PTPase activity in skeletal muscle fractions from insulin resistant rodents and we and others found decreased PTPase activity in muscle fractions from Type II diabetic patients [4-6]. Because of the focus on PTPase $1 \mathrm{~B}$ we extended our human studies [5] to measurements of PTPase 1B protein in skeletal muscle fractions from eight Type II diabetic patients and nine control subjects before and after a 3 h-hyperinsulinaemic, euglycaemic clamp study. Biopsies were taken from the vastus lateralis muscle during the earlier study [5].

The intensity of the band of PTPase 1B protein was decreased in Type II diabetic patients compared with control subjects in the basal state $[0.42 \pm 0.08$ vs $0.74 \pm 0.13$ (arbitrary units), $p<0.04]$. In control subjects the intensity of the band was increased after $3 \mathrm{~h}$ hyperinsulinaemia $(0.74 \pm 0.13$ vs $1.31 \pm 0.22, p<0.05$ ) but the seemingly positive effect of hyperinsulinaemia was not statistically significant in Type II diabetic patients $(0.42 \pm 0.08$ vs $0.73 \pm 0.10$, NS $)$.

These results confirm previous findings that PTPase $1 \mathrm{~B}$ protein was reduced in muscle fractions from Type II diabetic patients $[4,6]$. The decreased PTPase $1 \mathrm{~B}$ protein may fully or partly be responsible for the decreased PTPase activity in muscle fractions from these patients. The decreased PTPase activity in skeletal muscle fractions from Type II diabetic patients [4-6] may be a compensatory mechanism facilitating insulin signalling.

From the above it seems without doubt that PTPase 1B (ubiquitously and abundantly expressed) is important in the regulation of the intracellular insulin receptor signalling. It is possible that PTPase $1 \mathrm{~B}$ is a useful target for treatment with drugs in certain types of insulin resistance. The intracellular regulation of PTPase activity in insulin resistant states seems, 


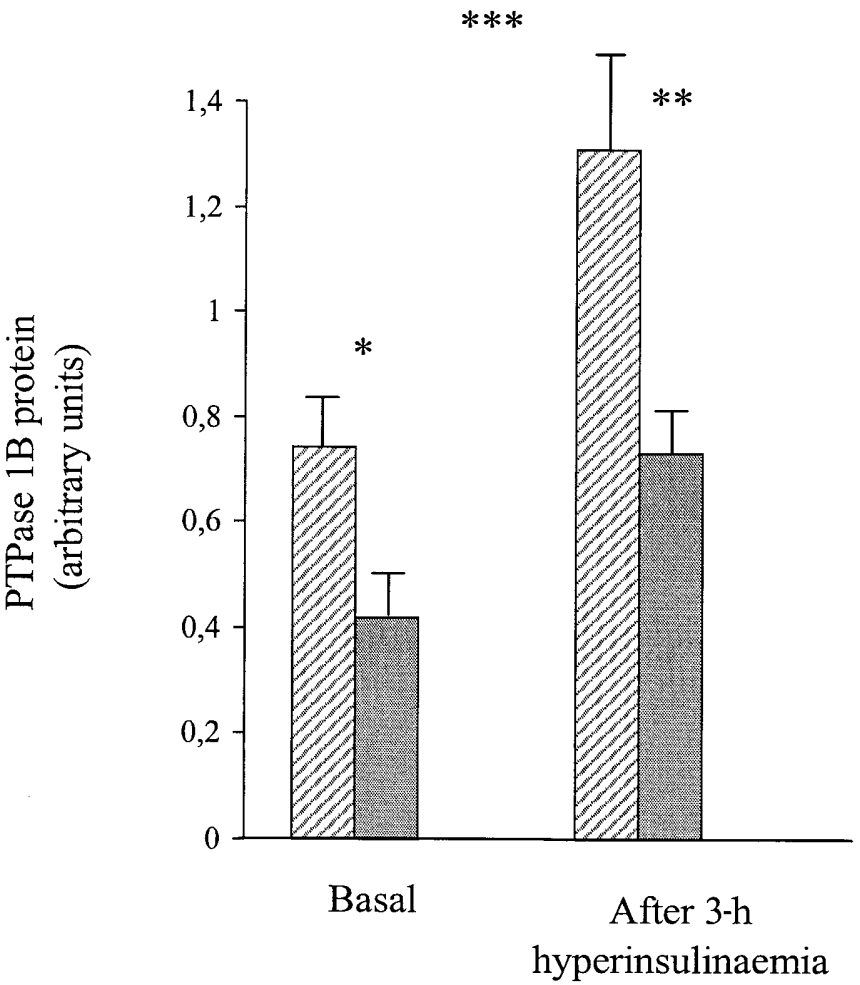

Fig. 1. PTPase 1B protein (arbitrary units) in particulate muscle fractions. Fractions were prepared from muscle biopsies taken before and after a 3 h-hyperinsulinaemic clamp from eight Type II diabetic patients and nine control subjects. Equal amounts of protein in muscle fractions were analysed by SDSPAGE (10\%) and Western blotting. Monoclonal mouse-antiPTP1B was from Upstate Biotechnology Incorporated (Lake Placid, N. Y., USA). Several bands were detected in particulate muscle fractions. A distinct band corresponding to PTPase 1B $(50 \mathrm{kDa})$ was quantified by densitometric scanning. This band was not detectable in soluble and cytoskeletal fractions. The results are the average of scanning of eight immunoblots. Groups of unpaired data were compared by means of the Mann-Whitney test, groups of paired data by means of the Wilcoxon test. $P$-values less than 0.05 were considered significant. WA control subjects, Type II diabetic patients. * $p<0.04$, control subjects vs Type II diabetic patients before the hyperinsulinaemic clamp. ${ }^{* *} \mathrm{p}=0.05$, control subjects vs Type II diabetic patients after the hyperinsulinaemic clamp. $* * * p<0.05$, control subjects before vs after the hyperinsulinaemic clamp

however, more complex than often indicated. The PTPase-mediated inhibition of the insulin receptor phosphorylation appears to be low in Type II diabetic patients compared with insulin sensitive subjects. Thus, to inhibit PTPase activity in Type II diabetic patients could be an attempt to push a compensatory process beyond its natural limit. Moreover, it has been found that PTPase $1 \mathrm{~B}$ activity and protein increased in muscle cells after insulin stimulation [7] and in another report it was found that the increased activity followed tyrosine phosphorylation of PTPase 1B by the insulin receptor kinase [8]. It could be suggested that these results, which agree with the present increase in PTPase 1B protein after hyperinsulinaemia in insulin sensitive control subjects, represent an important amplification-control/resetting mechanism, necessary for optimal timing of insulin signalling. In accordance with this, our laboratory found that the PTPase activity increased in particulate muscle fractions from insulin sensitive subjects after insulin stimulation, followed by a decrease in activity (unpublished data).

Importantly, human studies on PTPase activity and PTPase 1B protein in skeletal muscle are not considered in the paper by Elchebly et al. [1]. In our view, a more thorough understanding of the role of PTPase $1 \mathrm{~B}$ in the regulation of the insulin signalling cascade, which takes into account available findings, will be useful in the future development of PTPase 1Bspecific inhibitors as drugs for treatment of insulin resistance.

Yours sincerely,

D. Worm, J. Vinten, H. Beck-Nielsen

\section{References}

1. Elchebly M, Payette P, Michaliszyn E et al. (1999) Increased insulin sensitivity and obesity resistance in mice lacking the protein tyrosine phosphatase-1B gene. Science 283: $1544-1548$

2. Josefson D (1999) Scientists discover a gene involved in diabetes and obesity. BMJ 318: 689

3. McGuire MC, Fields RM, Nyomba BL et al. (1991) Abnormal regulation of protein tyrosine phosphatase activities in skeletal muscle of insulin-resistant humans. Diabetes 40 : 939-942

4. Kusari J, Kenner KA, Suh K-I, Hill DE, Henry RR (1994) Skeletal muscle protein tyrosine phosphatase activity and tyrosine phosphatase $1 \mathrm{~B}$ protein content are associated with insulin action and resistance. J Clin Invest 93: 1156-1162

5. Worm D, Vinten J, Stæhr P, Henriksen JE, Handberg A, Beck-Nielsen H (1996) Altered basal and insulin-stimulated phosphotyrosine phosphatase (PTPase) activity in skeletal muscle fractions from NIDDM patients compared with control subjects. Diabetologia 39: 1208-1214

6. Ahmad F, Azevedo JL, Cortright R, Dohm GL, Goldstein BJ (1997) Alterations in skeletal muscle protein-tyrosine phosphatase activity and expression in insulin-resistant human obesity and diabetes. J Clin Invest 100: 449-458

7. Kenner KA, Hill DE, Olefsky JM, Kusari J (1993) Regulation of protein tyrosine phosphatases by insulin and insulinlike growth factor 1. J Biol Chem 278: 25455-25462

8. Kusari J, Bandhyopadhay D, Kenner K, Kusari A (1997) Activated insulin receptor phosphorylates and increases the catalytic activity of protein tyrosine phosphatase $1 \mathrm{~B}$ (PTPase 1B) (Abstract). Diabetes 46:[Suppl 1] 204A 